\title{
3 Researc Square

\section{Cystectomy for Patients With Hunner-Type Interstitial Cystitis at a Tertiary Referral Center in Japan}

Yoshiyuki Akiyama ( $\sim$ yakiyamauro-tky@umin.ac.jp )

The University of Tokyo

Aya Niimi

New Tokyo Hospital

Yasuhiko Igawa

Nagano Prefectural Shinshu Medical Center

Akira Nomiya

National Center for Global Health and Medicine

Yusuke Sato

The University of Tokyo

\section{Yuta Yamada}

The University of Tokyo

Haruki Kume

The University of Tokyo

\section{Yukio Homma}

Japanese Red Cross Medical Center

\section{Research Article}

Keywords: interstitial cystitis, bladder pain syndrome, cystectomy, IC/BPS, PBS/IC, Hunner, augmentation

Posted Date: June 3rd, 2021

DOI: https://doi.org/10.21203/rs.3.rs-571569/v1

License: (c) (1) This work is licensed under a Creative Commons Attribution 4.0 International License. Read Full License

Version of Record: A version of this preprint was published at LUTS: Lower Urinary Tract Symptoms on October 26th, 2021. See the published version at https://doi.org/10.1111/luts.12416. 


\section{Abstract}

Cystectomy is an ultimate treatment option for patients with end-stage Hunner-type interstitial cystitis (HIC). However, pertinent information regarding this procedure such as optimal candidate selection, surgical procedures, possible complications, and treatment prognosis, is limited owing to its extremely low prevalence. We report outcomes of 17 cystectomies for end-stage HIC patients at a tertiary referral center in Japan over a period of 20 years, including partial cystectomy with augmentation ileocystoplasty (PC-CP, 4) and total cystectomy with ileal conduit (TC-IC, 13). Postoperative events including changes in symptoms and quality of life (QOL), related complications, and overall satisfaction were examined. Pain persisted in three PC-CP patients while completely resolved in all TC-IC patients. QOL improved significantly in TC-IC but not in PC-CP patients. Two PC-CP patients required clean intermittent catheterization due to voiding dysfunction. Two TC-IC patients developed stricture of ureteroileal anastomosis, resulting in permanent placement of a ureteral stent or nephrostomy. Satisfaction rate was significantly higher in TC-IC than in PC-CP patients $(76.9 \%$ vs $25.0 \%, p<0.05)$. Despite the limited number of cases, this study demonstrates that TC-IC provides highly reliable pain relief and improves QOL in end-stage HIC patients.

\section{Introduction}

Hunner-type interstitial cystitis (HIC) is a chronic inflammatory disease of the urinary bladder of unknown etiology, clinically characterized by persistent pelvic pain and lower urinary tract symptoms such as urinary frequency and urgency. ${ }^{1}$ The progressive nature of this disease in some patients can result in severe bladder contraction, leading to incredibly increased urinary frequency and/or urinary leakage. Associated vesicoureteral reflux (VUR) may cause hydronephrosis, recurring pyelonephritis, or irreversible renal dysfunction. Cystectomy may be the ultimate treatment for patients with end-stage HIC, as described in clinical guidelines for patients with interstitial cystitis/bladder pain syndrome (IC/BPS). ${ }^{12} 3$ Because this procedure is rarely performed, however, detailed information on cystectomy, such as candidate selection, surgical procedures, possible complications, and treatment prognosis, is limited. The present study describes our 20 years' experience with 17 patients who underwent subtrigonal partial cystectomy with augmentation ileocystoplasty $(\mathrm{PC}-\mathrm{CP})$ or total cystectomy with ileal conduit $(\mathrm{TC}-\mathrm{IC})$ for end-stage HIC.

\section{Methods}

\section{Ethics statement}

The Institutional Review Board of the University of Tokyo approved the study protocol, including the use of an opt-out methodology to obtain informed consent (approval no. 3124). Participants were informed about the study using generally accessible contact information. Written informed consent was provided by participants who chose to take part in this study. All procedures followed appropriate guidelines. 


\section{Patients}

The clinical database of the University of Tokyo Hospital was retrospectively reviewed to identify patients with end-stage HIC who underwent PC-CP or TC-IC between 2002 and 2020. Diseases were diagnosed and classified according to East Asian and European clinical guidelines for IC/BPS. ${ }^{118}$

\section{Surgical procedures}

Cystectomy was considered for patients with end-stage HIC if their QOL was markedly impaired or if they had experienced significant complications in the upper urinary tract, such as persistent hydronephrosis and/or recurrent (defined as three times or more per year) pyelonephritis. The former was characterized by intractable and persistent bladder pain, extraordinarily frequent urination, or continued urinary leakage that could not be controlled by an indwelling urethral catheter. A thorough personal consultation was performed prior to surgery. Patients were informed of related risks, such as persistence/recurrence of symptoms, the need for $\mathrm{CIC}$, the irreversibility of the procedure and body image of the stoma, and the risk of perioperative complications. All patients provided informed consent for cystectomy and the procedure, PC-CP or TC-IC.

The PC-CP procedure involved excision of the bladder wall while preserving the bladder neck and trigonal area with bladder augmentation using ileal segments. The TC-IC procedure consisted of simple cystectomy and ileal conduit formation, with urethrectomy in male patients, as indicated. TC-NB was not attempted because of the higher risks of complications and postoperative CIC.

Postoperative complications in both groups were monitored by assessing symptoms, by performing blood tests and urinalysis, by urine culture, by measurements of post-void residual urine, and by ultrasound scanning. Postoperative complications were managed by additional interventions, as needed. All operations were performed using an open approach by one of the four specialized urologists (YA, ANi, $\mathrm{YI}, \mathrm{YH})$ at our facility.

\section{Assessment and evaluation}

Symptoms were assessed using an 11-point numerical rating of pain intensity, with 0 indicating no pain and 10 indicating the worst pain imaginable. QOL scores were measured on a 7-grade QOL scale derived from the International Prostate Symptom Score, with 0 indicating an excellent QOL and 6 indicating a terrible QOL. OSSI/OSPI indices were assessed pre- and postoperatively in patients who underwent PC$\mathrm{CP}$ alone, as questions on voiding frequency and urgency are inapplicable to patients who undergo TCIC. Patients were closely monitored for anticipated complications, such as voiding difficulty necessitating $\mathrm{CIC}$, hydronephrosis, and pyelonephritis. Hydronephrosis was graded according to the Society for Fetal Urology ultrasound grading system. ${ }^{19}$ Patients' overall satisfaction with surgical outcomes was assessed using a yes-or-no question 1 year after surgery or when complications were stabilized.

\section{Statistical analysis}


Symptom parameters before and after surgery in each group were compared by Wilcoxon signed rank tests, whereas symptom parameters in the two groups were compared by Wilcoxon rank sum tests. Categorical variables were compared using Fisher's exact tests. All statistical analyses were performed using JMP® software, version 14 (SAS Institute, Cary, NC, USA), with $P$-values $<0.05$ considered statistically significant.

\section{Results}

A retrospective review of the clinical database of our institution identified 17 patients with end-stage HIC who underwent cystectomy between 2002 and 2020. Four patients, three men and one woman, underwent PC-CP, whereas 13 patients, nine women and four men, underwent TC-IC. Treatments before cystectomy included oral analgesic drugs, such as nonsteroidal anti-inflammatory drugs (NSAIDs), pregabalin, and opioids; anti-cholinergic agents; beta-3 adrenoceptor agonists; tricyclic antidepressants such as amitriptyline; anti-allergic agents such as suplatast tosilate, hydroxyzine, and cimetidine; oral steroids; intravesical instillation, including with dimethyl sulfoxide, alkalized heparin and lidocaine, and botulinum toxin; and electrocautery of Hunner lesions with hydrodistension.

The pre- and perioperative demographic characteristics of these patients are shown in Table 1. Mean overall follow-up time was $4.7 \pm 3.6$ years overall. At the time of surgery, patients undergoing PC-CP were significantly younger (mean age, $53.5 \pm 12.9$ years) than those with TC-IC $(67.0 \pm 10.9$ years). Duration of illness, O'Leary and Sant's Symptom (OSSI) and Problem (OSPI) scores, pain intensity, quality of life (QOL) scores, daytime frequency, nocturia, maximum voided volume (MVV) on the frequency volume chart, maximum bladder capacity (MBC) at the last hydrodistension (under a pressure of $80 \mathrm{~cm} \mathrm{H}_{2} \mathrm{O}$ ), and the total number of previous sessions of Hunner lesion electrocautery were comparable in these two groups. Operation time, blood loss, and transfusion rate were higher, however, in the TC-IC than in the PC-CP group. 
Table 1

Demographic and clinical characteristics of patients who underwent cystectomy for Hunner-type interstitial cystitis

\begin{tabular}{|c|c|c|c|}
\hline & $\mathrm{PC}-\mathrm{CP}$ & TC-IC & $p$-value \\
\hline No. of patients (female/male) & $4(1 / 3)$ & $13(9 / 4)$ & \\
\hline Age at surgery (y) & $53.5 \pm 12.9[35-65]$ & $67.0 \pm 10.9[39-79]$ & $<0.05^{\star}$ \\
\hline Duration of illness $(y)$ & $10.0 \pm 8.2[2-21]$ & $8.3 \pm 4.9[3-18]$ & 0.78 \\
\hline Postoperative follow-up period (y) & $5.3 \pm 5.9[1-12]$ & $4.5 \pm 3.2[1-11]$ & 0.95 \\
\hline OSSI & $17.3 \pm 2.5[15-20]$ & $15.1 \pm 3.8[8-19]$ & 0.34 \\
\hline OSPI & $14.1 \pm 2.0[10-16]$ & $13.1 \pm 3.8[4-16]$ & 0.99 \\
\hline Pain scale ${ }^{\ddagger}$ & $8.5 \pm 1.0[8-10]$ & $7.4 \pm 2.1[2-9]$ & 0.45 \\
\hline QOL score & $5.5 \pm 0.6[5-6]$ & $5.7 \pm 0.9[3-6]$ & 0.25 \\
\hline Daytime frequency & $22.3 \pm 6.8[17-30]$ & $18.0 \pm 3.9[13-25]$ & 0.24 \\
\hline Nocturia & $6.3 \pm 6.7[2-14]$ & $4.4 \pm 1.5[2-8]$ & 0.57 \\
\hline Maximum voided volume $(\mathrm{mL})$ & $\begin{array}{l}87.5 \pm 22.2[60- \\
110]\end{array}$ & $69.2 \pm 23.9[30-110]$ & 0.18 \\
\hline $\begin{array}{l}\text { Maximum bladder capacity } \\
\text { at the last hydrodistension }(\mathrm{mL})\end{array}$ & $\begin{array}{l}162.5 \pm 85.0[80- \\
250]\end{array}$ & $\begin{array}{l}237.5 \pm 161.1[100- \\
300]\end{array}$ & $<0.33$ \\
\hline $\begin{array}{l}\text { No. of previous electrocautery sessions for } \\
\text { Hunner lesions }\end{array}$ & $3.3 \pm 1.0[2-4]$ & $3.8 \pm 2.0[1-8]$ & 0.62 \\
\hline Grade of hydronephrosis (no. of pts) & & & 0.58 \\
\hline Grade 0 & 3 & 6 & \\
\hline Grade 1 & 0 & 1 & \\
\hline Grade 2 & 1 & 2 & \\
\hline
\end{tabular}

Results are reported as mean \pm SD [range], unless otherwise indicated.

Abbreviations: PC-CP, supratrigonal partial cystectomy with ileocystoplasty; TC-IC, total cystectomy with ileal conduit; OSSI, O'Leary and Sant's Symptom Index; OSPI, O'Leary and Sant's Problem Index; QOL, quality of life.

${ }^{*} p<0.05$, statistically significant.

${ }^{\ddagger}$ Assessed using an 11-point pain intensity numerical rating scale from 0 , indicating no pain, to 10 , indicating the worst pain imaginable.

'Classified using the Society for Fetal Urology ultrasound grading system of hydronephrosis (ref. 19). 


\begin{tabular}{|c|c|c|c|}
\hline & $\mathrm{PC}-\mathrm{CP}$ & TC-IC & $p$-value \\
\hline Grade 3 & 0 & 2 & \\
\hline Grade 4 & 0 & 2 & \\
\hline $\begin{array}{l}\text { Increases in serum creatinine level (no. of } \\
\text { pts) }\end{array}$ & 0 & 5 & 0.26 \\
\hline Operation time (min) & $\begin{array}{l}261.0 \pm 48.1[220- \\
314]\end{array}$ & $\begin{array}{l}401.8 \pm 70.1[328- \\
531]\end{array}$ & $<0.01^{*}$ \\
\hline Blood loss (mL) & $\begin{array}{l}886.7 \pm 496.9 .1 \\
{[580-1460]}\end{array}$ & $\begin{array}{l}1795.0 \pm 1024.0 \\
{[555-3615]}\end{array}$ & 0.23 \\
\hline Transfusion (no. of pts) & 0 & 7 & $<0.05^{\star}$ \\
\hline \multicolumn{4}{|c|}{ Results are reported as mean \pm SD [range], unless otherwise indicated. } \\
\hline \multicolumn{4}{|c|}{$\begin{array}{l}\text { Abbreviations: PC-CP, supratrigonal partial cystectomy with ileocystoplasty; TC-IC, total cystectomy } \\
\text { with ileal conduit; OSSI, O'Leary and Sant's Symptom Index; OSPI, O'Leary and Sant's Problem Index; } \\
\text { QOL, quality of life. }\end{array}$} \\
\hline \multicolumn{4}{|l|}{${ }^{*} p<0.05$, statistically significant. } \\
\hline \multicolumn{4}{|c|}{$\begin{array}{l}{ }^{\ddagger} \text { Assessed using an } 11 \text {-point pain intensity numerical rating scale from } 0 \text {, indicating no pain, to } 10 \text {, } \\
\text { indicating the worst pain imaginable. }\end{array}$} \\
\hline
\end{tabular}

Table 2 displays postoperative outcomes in these patients. Three (75\%) of the four patients who underwent $\mathrm{PC}-\mathrm{CP}$ experienced recurrent pain within 15 months after surgery, compared with none $(0 \%)$ of the 13 patients who underwent TC-IC. Removal or preservation of the distal urethra was not associated with postoperative symptoms in men undergoing TC-IC (data not shown). Pain intensity and QOL scores were significantly improved in patients who underwent TC-IC, whereas pain intensity, QOL scores, and OSSI/OSPI scores were not significantly improved in patients who underwent PC-CP (Fig. 1). Three $(75 \%)$ of the four patients in the PC-CP group started clean intermittent catheterization (CIC) within 18 months after surgery, two (Patient nos. 2 and 3 ) due to voiding dysfunction and one (Patient no. 1) due to recurrent severe pain during urine storage. Two patients who underwent TC-IC experienced stricture of the ureteroileal anastomosis with hydronephrosis and/or pyelonephritis: one (Patient no. 7) was managed by installation of a permanent ureteral stent, and the other (Patient no. 10) by nephrostomy. Preoperative Grade 2 hydronephrosis (without an increase in serum creatinine levels) resolved after surgery in one patient who underwent PC-CP. Seven patients in the TC-IC group had preoperative hydronephrosis, including one with Grade 1 and two each with Grades 2, 3, and 4), with five showing increased serum creatinine concentrations. Of these five patients, four showed normalization of creatinine levels after surgery, whereas one (Patient no. 10) developed postoperative ureteroileal 
anastomosis stricture and required nephrostomy replacement. Satisfaction with overall surgical outcomes was significantly higher in the TC-IC than in the PC-CP group (76.9\% vs. $25.0 \%, p<0.05)$. 
Table 2

Postoperative events

\begin{tabular}{|c|c|c|c|c|c|}
\hline $\begin{array}{l}\text { Patient } \\
\text { no. } \\
\text { (age, } \\
\text { sex) }\end{array}$ & Procedure & $\begin{array}{l}\text { Recurrent } \\
\text { pain } \\
(\mathrm{mo})^{\dagger}\end{array}$ & $\begin{array}{l}\text { QOL } \\
\text { score }\end{array}$ & Satisfaction & Complications/Note \\
\hline $\begin{array}{l}1(65, \\
M)\end{array}$ & $\mathrm{PC}-\mathrm{CP}$ & Yes (15) & 5 & No & $\mathrm{CIC}(18 \mathrm{mo})^{\dagger}$ \\
\hline $\begin{array}{l}2(58 \\
M)\end{array}$ & $\mathrm{PC}-\mathrm{CP}$ & Yes (3) & 4 & No & CIC (3 mo) \\
\hline $\begin{array}{l}3(35 \\
F)\end{array}$ & $\mathrm{PC}-\mathrm{CP}$ & No & 3 & Yes & CIC (6 mo) \\
\hline $\begin{array}{l}4(56 \\
M)\end{array}$ & $\mathrm{PC}-\mathrm{CP}$ & Yes (2) & 6 & No & None \\
\hline $\begin{array}{l}5(76 \\
F)\end{array}$ & $\mathrm{TC}-\mathrm{IC}$ & No & 0 & Yes & None \\
\hline $\begin{array}{l}6(73 \\
M)\end{array}$ & TC-IC & No & 1 & Yes & None \\
\hline $\begin{array}{l}7(73 \\
M)\end{array}$ & TC-IC & No & 5 & No & $\begin{array}{l}\text { Ureteroileal anastomosis }(2 \mathrm{wk}) \text {, ureteral } \\
\text { stent replacement }(6 \mathrm{mo}) \text {, pyelonephritis } \\
(6 \mathrm{mo})\end{array}$ \\
\hline $\begin{array}{l}8(64 \\
M)\end{array}$ & TC-IC & No & 0 & Yes & None \\
\hline $\begin{array}{l}9(73 \\
M)\end{array}$ & TC-IC & No & 0 & Yes & None \\
\hline $\begin{array}{l}10(69, \\
F)\end{array}$ & TC-IC & No & 6 & No & $\begin{array}{l}\text { Pyelonephritis }(15 \mathrm{mo}) \text {, ureteroileal } \\
\text { anastomosis }(21 \mathrm{mo}) \text {, nephrostomy } \\
\text { replacement ( } 36 \mathrm{mo})\end{array}$ \\
\hline $\begin{array}{l}11(54, \\
F)\end{array}$ & TC-IC & No & 0 & Yes & None \\
\hline $\begin{array}{l}12(75, \\
\text { F) }\end{array}$ & TC-IC & No & 0 & Yes & None \\
\hline $\begin{array}{l}13(65, \\
\text { F) }\end{array}$ & TC-IC & No & 0 & Yes & None \\
\hline $\begin{array}{l}14(39, \\
\text { F) }\end{array}$ & TC-IC & No & 0 & Yes & Adhesive ileus (14 d) \\
\hline
\end{tabular}

Abbreviations: PC-CP, supratrigonal partial cystectomy with ileocystoplasty; TC-IC, total cystectomy with ileal conduit; $\mathrm{CIC}$, clean intermittent catheterization; QOL, quality of life.

${ }^{\dagger}$ Time from surgery. 


\begin{tabular}{|c|c|c|c|c|c|}
\hline $\begin{array}{l}\text { Patient } \\
\text { no. } \\
\text { (age, } \\
\text { sex) }\end{array}$ & Procedure & $\begin{array}{l}\text { Recurrent } \\
\text { pain } \\
(\mathrm{mo})^{\dagger}\end{array}$ & $\begin{array}{l}\text { QOL } \\
\text { score }\end{array}$ & Satisfaction & Complications/Note \\
\hline $\begin{array}{l}15(61, \\
F)\end{array}$ & TC-IC & No & 0 & Yes & None \\
\hline $\begin{array}{l}16(79 \\
F)\end{array}$ & TC-IC & No & 0 & Yes & None \\
\hline $\begin{array}{l}17(71, \\
F)\end{array}$ & TC-IC & No & 1 & Yes & None \\
\hline \multicolumn{6}{|c|}{$\begin{array}{l}\text { Abbreviations: PC-CP, supratrigonal partial cystectomy with ileocystoplasty; TC-IC, total cystectomy } \\
\text { with ileal conduit; CIC, clean intermittent catheterization; QOL, quality of life. }\end{array}$} \\
\hline${ }^{\dagger}$ Time $\mathrm{f}$ & surgery. & & & & \\
\hline
\end{tabular}

\section{Discussion}

The present study reports outcomes in 17 consecutive patients who underwent cystectomy for end-stage HIC at a tertiary referral center for IC/BPS in Japan. Although TC-IC was a more invasive procedure and more likely to cause postoperative complications than PC-CP, TC-IC was able to achieve almost complete pain relief and a higher rate of patient satisfaction.

Endoscopic electrocautery of Hunner lesions with bladder hydrodistension is regarded as the most promising treatment for HIC. ${ }^{45}$ However, intractable bladder pain and associated lower urinary tract symptoms may persist even after repeated sessions of electrocautery. ${ }^{6}$ Some patients progressively develop contracted bladder, which frequently induces VUR and/or hydronephrosis. Cystectomy may be an option for these patients with end-stage HIC. ${ }^{123}$ To date, however, optimal patient selection and standardized surgical procedures have not been established.

Our center regards patients with markedly impaired QOL resulting from intractable bladder pain or extremely severe bladder storage symptoms, or upper urinary tract events such as VUR, persistent hydronephrosis, and recurrent pyelonephritis, as candidates for cystectomy. Because TC is likely to provide greater symptom relief than PC, 78910 we basically propose TC for the surgical approach of choice. For example, complete pain resolution was achieved by nine (90\%) of 10 women after $\mathrm{TC}^{9}$, with a patient satisfaction rate $>77 \%$ despite the high complication rate $(60 \%)$. By contrast, persistent bladder pain was experienced by eight (33\%) of 24 patients ${ }^{7}$ and by six (75\%) of eight patients ${ }^{8}$ after PC. Furthermore, persistent/recurrent pain was reported in five (17\%) of 29 patients who underwent PC-CP, compared with none $(0 \%)$ of six patients who underwent TC-IC. ${ }^{10}$ Meanwhile, several studies have found that PC-CP has modest success rates in patients with refractory IC/BPS. ${ }^{11} 1213$ For example, PC$\mathrm{CP}$ had a success rate of $80 \%$ in 40 patients with $\mathrm{HIC}$, with five patients requiring $\mathrm{CIC}$ during a median 
follow-up of 12.0 months. ${ }^{11} \mathrm{~A}$ study of 17 patients who underwent $\mathrm{TC}$ and six who underwent PC with orthotopic bladder substitution using an ileocecal pouch (Mainz pouch $\Downarrow$ ) found that none of the PC patients developed pain recurrence, compared with one TC patient who experienced persistent pain after surgery. ${ }^{12} \mathrm{~A}$ recent meta-analysis reviewing 20 articles demonstrated that symptom improvement rates were $90.5 \%$ for TC overall (94.9\% for TC with neo-bladder formation, TC-NB and $82 \%$ for TC and any diversion), $73.4 \%$ for $\mathrm{PC}-\mathrm{CP}$, and $63.4 \%$ for diversion alone. ${ }^{14}$ The overall complication rate was $26.5 \%$, with TC-NB having the highest and TC-IC having the lowest complication rate.

In our facility, cystectomy was not performed in IC/BPS patients without Hunner lesions due to the low expectancy of success. A study reporting the outcomes of various reconstructive surgeries in patients with IC/BPS found that success rates were much higher in patients with $\mathrm{HIC}(>80 \%)$ than in patients without Hunner lesions (23\%). ${ }^{15}$ Moreover, PC-CP resulted in symptom resolution in all $10 \mathrm{HIC}$ patients but in none of the three patients without Hunner lesions. ${ }^{16}$ Recent evidence revealed that HIC is a robust inflammatory disease of the bladder whereas other forms of IC/BPS that lack Hunner lesions are noninflammatory disorders, potentially involving psychological and/or neuropathological conditions outside the bladder. ${ }^{17}$ Thus, cystectomy outcomes should favor HIC which affects the bladder.

The present study has several limitations, including its retrospective study design and small sample size. In particular, this study was essentially limited to patients who underwent TC-IC, as only four underwent $\mathrm{PC}-\mathrm{CP}$ and none underwent neo-bladder formation. Further studies are needed to determine the surgical outcomes of cystectomy for refractory IC/BPS and to identify factors prognostic of success, thereby optimizing patient selection and surgical procedures.

In conclusion, TC-IC provided highly reliable pain relief and patient satisfaction in patients with endstage HIC. Further studies are warranted to identify appropriate candidates and optimal surgical procedures for patients with refractory IC/BPS.

\section{Abbreviations}

BPS = bladder pain syndrome

$\mathrm{CIC}=$ clean intermittent catheterization

ESSIC = International Society for the Study of BPS

HIC = Hunner type interstitial cystitis

IC = interstitial cystitis

$\mathrm{MVV}=$ maximum voided volume

$\mathrm{MBC}=$ maximum bladder capacity 
NSAID = nonsteroidal anti-inflammatory drug

OSSI/OSPI = O'Leary and Sant's Symptom and Problem Indices

$\mathrm{QOL}$ = quality of life

$\mathrm{PC}-\mathrm{CP}=$ supratrigonal partial cystectomy with ileocystoplasty

$\mathrm{TC}-\mathrm{IC}=$ total cystectomy with ileal conduit

$\mathrm{TC}-\mathrm{NB}=$ total cystectomy with neo-bladder

VUR = vesicoureteral reflux

\section{Declarations}

\section{Acknowledgments}

The authors thank the study participants.

\section{Grants}

This study was supported by a KAKENHI Grant-in-Aid to YA from the Japan Society for the Promotion of Science (JSPS) (grant number: 19K18552) and by a Health Labour Sciences Research Grant to YH from the Ministry of Health, Labour, and Welfare (grant number: 18060798).

\section{Disclosure statement}

All authors declare no conflicts of interest, financial or otherwise, regarding this study.

\section{Consent to participate}

Participants were informed about the study using generally accessible contact information, and written informed consent was obtained from participants who chose to participate.

\section{Consent for publication}

All authors consent to the publication of the manuscript, should the article be accepted by the Editor-inChief upon completion of the refereeing process. All participants consent to the publication of the study in a journal, website, or other form of publication.

\section{Availability of data and material}

The data that support the findings of this study are available from the corresponding author upon reasonable request. 


\section{Author contributions}

$\mathrm{YA}$ and $\mathrm{ANi}$ collected the data. $\mathrm{YA}, \mathrm{ANi}, \mathrm{YI}$, and $\mathrm{YH}$ performed the operations. $\mathrm{YA}$ and $\mathrm{ANi}$ analyzed the data. YA and $\mathrm{YH}$ wrote the manuscript. $\mathrm{YI}, \mathrm{AN}, \mathrm{YS}, \mathrm{YY}, \mathrm{HK}$, and $\mathrm{YH}$ revised the manuscript critically. $\mathrm{YA}$ and $\mathrm{YH}$ provided final approval of the version to be submitted.

\section{References}

1. Homma, Y. et al. Clinical guidelines for interstitial cystitis/bladder pain syndrome. International journal of urology: official journal of the Japanese Urological Association, doi:10.1111/iju.14234 (2020).

2. Hanno, P. M., Erickson, D., Moldwin, R., Faraday, M. M. \& American Urological, A. Diagnosis and treatment of interstitial cystitis/bladder pain syndrome: AUA guideline amendment. The Journal of urology 193, 1545-1553, doi:10.1016/j.juro.2015.01.086 (2015).

3. Cox, A. et al. CUA guideline: Diagnosis and treatment of interstitial cystitis/bladder pain syndrome. Canadian Urological Association journal = Journal de l'Association des urologues du Canada 10, E136-E155, doi:10.5489/cuaj.3786 (2016).

4. Ko, K. J. et al. Comparison of the Efficacy Between Transurethral Coagulation and Transurethral Resection of Hunner Lesion in Interstitial Cystitis/Bladder Pain Syndrome Patients: A Prospective Randomized Controlled Trial. European urology, doi:10.1016/j.eururo.2020.01.002 (2020).

5. Chennamsetty, A. et al. Electrosurgical management of Hunner ulcers in a referral center's interstitial cystitis population. Urology 85, 74-78, doi:10.1016/j.urology.2014.09.012 (2015).

6. Akiyama, Y. et al. Relationship between the frequency of electrocautery of Hunner lesions and changes in bladder capacity in patients with Hunner type interstitial cystitis. Scientific reports 11, 105, doi:10.1038/s41598-020-80589-3 (2021).

7. Nurse, D. E., Parry, J. R. \& Mundy, A. R. Problems in the surgical treatment of interstitial cystitis. British journal of urology 68, 153-154 (1991).

8. Nielsen, K. K., Kromann-Andersen, B., Steven, K. \& Hald, T. Failure of combined supratrigonal cystectomy and Mainz ileocecocystoplasty in intractable interstitial cystitis: is histology and mast cell count a reliable predictor for the outcome of surgery? The Journal of urology 144, 255-258; discussion 258-259 (1990).

9. Peters, K. M., Jaeger, C., Killinger, K. A., Rosenberg, B. \& Boura, J. A. Cystectomy for ulcerative interstitial cystitis: sequelae and patients' perceptions of improvement. Urology 82, 829-833, doi:10.1016/j.urology.2013.06.043 (2013).

10. Mateu Arrom, L. et al. Long-term follow-up after cystectomy for bladder pain syndrome: pain status, sexual function and quality of life. World journal of urology, doi:10.1007/s00345-018-2554-6 (2018).

11. Kim, H. J. et al. Efficacy and safety of augmentation ileocystoplasty combined with supratrigonal cystectomy for the treatment of refractory bladder pain syndrome/interstitial cystitis with Hunner's 
lesion. International journal of urology: official journal of the Japanese Urological Association 21 Suppl 1, 69-73, doi:10.1111/iju.12320 (2014).

12. Linn, J. F. et al. Treatment of interstitial cystitis: comparison of subtrigonal and supratrigonal cystectomy combined with orthotopic bladder substitution. The Journal of urology $159,774-778$ (1998).

13. van Ophoven, A., Oberpenning, F. \& Hertle, L. Long-term results of trigone-preserving orthotopic substitution enterocystoplasty for interstitial cystitis. The Journal of urology 167, 603-607 (2002).

14. Osman, N. I. et al. A Systematic Review of Surgical interventions for the Treatment of Bladder Pain Syndrome/Interstitial Cystitis. European urology focus, doi:10.1016/j.euf.2020.02.014 (2020).

15. Rossberger, J., Fall, M., Jonsson, O. \& Peeker, R. Long-term results of reconstructive surgery in patients with bladder pain syndrome/interstitial cystitis: subtyping is imperative. Urology 70,638 642, doi:10.1016/j.urology.2007.05.028 (2007).

16. Peeker, R., Aldenborg, F. \& Fall, M. The treatment of interstitial cystitis with supratrigonal cystectomy and ileocystoplasty: difference in outcome between classic and nonulcer disease. The Journal of urology 159, 1479-1482, doi:10.1097/00005392-199805000-00018 (1998).

17. Akiyama, Y., Luo, Y., Hanno, P. M., Maeda, D. \& Homma, Y. Interstitial cystitis/bladder pain syndrome: The evolving landscape, animal models and future perspectives. International journal of urology: official journal of the Japanese Urological Association 27, 491-503, doi:10.1111/iju.14229 (2020).

18. van de Merwe, J. P. et al. Diagnostic criteria, classification, and nomenclature for painful bladder syndrome/interstitial cystitis: an ESSIC proposal. European urology 53, 60-67, doi:10.1016/j.eururo.2007.09.019 (2008).

19. Fernbach, S. K., Maizels, M. \& Conway, J. J. Ultrasound grading of hydronephrosis: introduction to the system used by the Society for Fetal Urology. Pediatr Radio/ 23, 478-480, doi:10.1007/BF02012459 (1993).

\section{Figures}


A

Pain

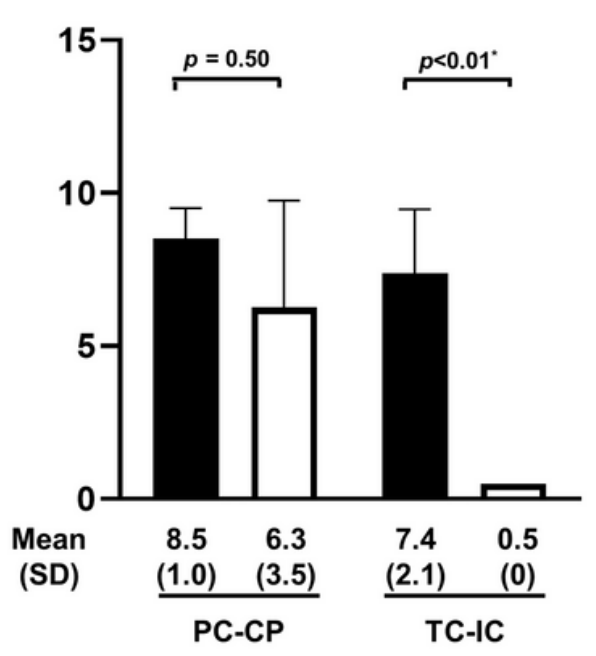

B

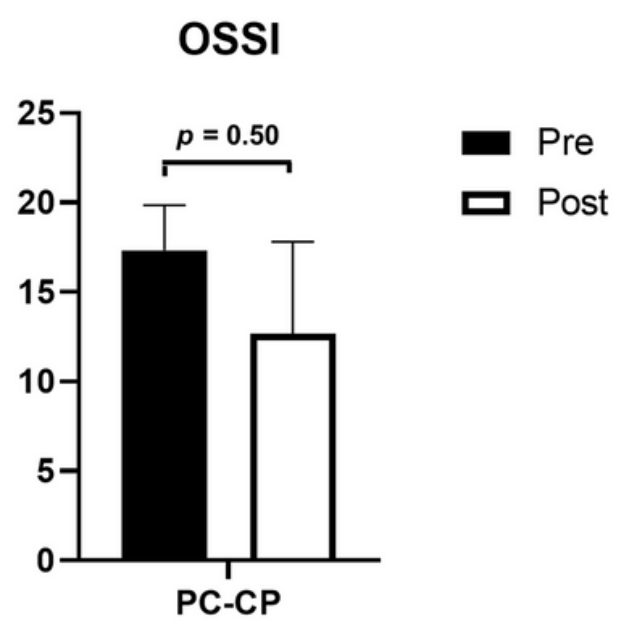

QOL

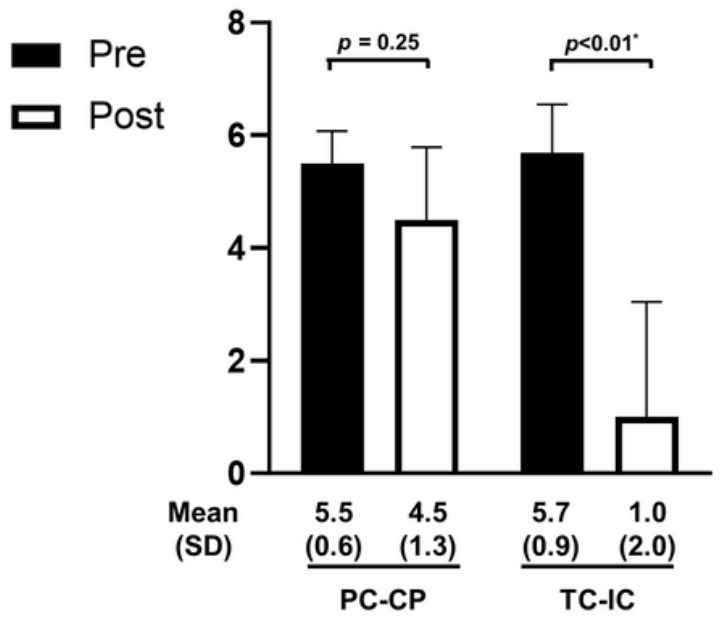

OSPI

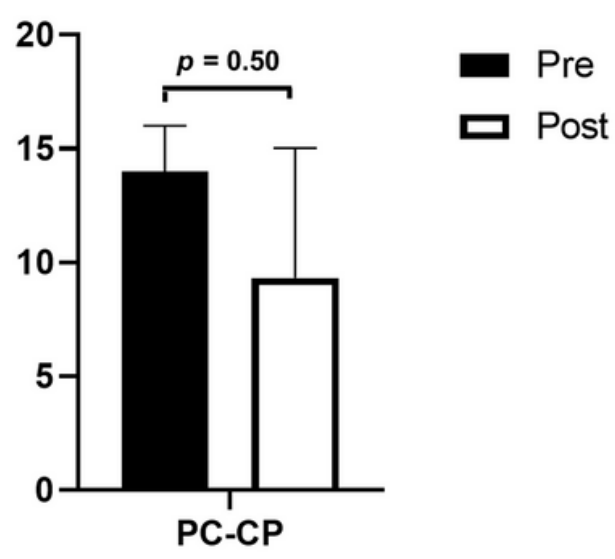

$\begin{array}{lll}\text { Mean } & 14.1 & 9.3\end{array}$

(SD) $\quad(2.0) \quad(5.7)$

Figure 1

Effects of TC-IC and PC-CP on patient outcomes. A: Changes in pain intensity and QOL scores. ${ }^{*} \mathrm{p}<0.05$ by Wilcoxon signed rank test. B: Changes in OSSI/OSPI scores in patients who underwent PC-CP. 